\title{
Physical activity of Korean cancer survivors is associated with age and sex
}

\author{
Jae Hyeon Park ${ }^{1}$, Jung Soo Lee ${ }^{2}$, Yoon $\mathrm{Ho} \mathrm{Ko}^{3}$, and Yeo Hyung Kim²
}

${ }^{1}$ Department of Rehabilitation Medicine, Hanyang University Guri Hospital, Guri; Departments of ${ }^{2}$ Rehabilitation Medicine and ${ }^{3}$ Internal Medicine, College of Medicine, The Catholic University of Korea, Seoul, Korea

Received: July 27, 2019

Revised : October 2, 2019

Accepted: November 4, 2019

\section{Correspondence to}

Yeo Hyung Kim, M.D.

Department of Rehabilitation Medicine, Uijeongbu St. Mary's Hospital, College of Medicine, The Catholic University of Korea, 271 Cheonbo-ro, Uijeongbu 11765, Korea

Tel: $+82-31-820-3172$

Fax: +82-31-847-8077

E-mail: drkyh@catholic.ac.kr https://orcid.org/0000-00023951-7205
Background/Aims: This study aimed to assess adherence to physical activity guidelines in cancer survivors and to investigate related factors.

Methods: The cross-sectional data of 39,845 community-dwelling participants aged $\geq 20$ years $(1,254$ cancer survivors $)$ in the Korea National Health and Nutrition Examination Survey were analyzed. The age- and sex-stratified prevalence for meeting exercise guidelines was obtained. The correlates of meeting exercise recommendations were evaluated by using complex-sample logistic regression models.

Results: Overall, $36.2 \%$ and $20.5 \%$ of cancer survivors met the recommendations for aerobic and resistance exercises, respectively. Cancer survivors and noncancer controls had similar low adherence to exercise recommendations in most age and sex groups, except young female cancer survivors ( 20 to 49 years old) who showed higher engagement in aerobic exercise than female noncancer controls in the same age group. Old cancer survivors ( $\geq 65$ years) were less likely to engage in recommended levels of aerobic exercise than young cancer survivors (odds ratio [OR], $0.44 ; 95 \%$ confidence interval [CI], 0.24 to 0.79 ). Female cancer survivors were less likely to meet resistance exercise guidelines than male cancer survivors (OR, 0.32; 95\% CI, 0.21 to 0.50 ). In addition, cancer survivors with current smoking status and cardiovascular disease were less likely to meet aerobic exercise recommendations.

Conclusions: The prevalence of meeting exercise guidelines are low in Korean cancer survivors and varied by age and sex. Old age is associated with an insufficient level of aerobic exercise, and female sex is associated with low participation in resistance exercise.

Keywords: Cancer survivors; Exercise; Physical fitness; Age groups; Sex

\section{INTRODUCTION}

The number of cancer survivors has increased due to the early detection of cancer and improvement of cancer treatment [1-3]. Cancer survivors have more comorbidities, such as cardiovascular diseases, chronic pain, and a low quality of life, than people without cancer [4-7]. Thus, lifestyle interventions for reducing cardiovascular disease and improving quality of life in cancer survivors have become an important issue. Physical activity (PA), including aerobic and resistance exercises, is a well-established lifestyle intervention for improving fatigue, quality of life, and body composition, and PA may reduce all-cause mortality in cancer survivors [8-11]. Given the multiple beneficial effects of PA in cancer survivors, the American College of Sports Medicine (ACSM) and Amer- 
ican Cancer Society (ACS) have recommended that cancer survivors should avoid physical inactivity and should perform regular aerobic and resistance exercises $[12,13]$.

Age and sex are crucial factors among the sociodemographic and health-related factors that can affect exercise compliance in the general population [14]. Nevertheless, in cancer survivors, there are limited data showing the impact of age and sex on adherence to PA recommendations. Several studies that investigated the associations between age or sex and participation in PA by cancer survivors have shown mixed observations [15-18]. Furthermore, although resistance exercise is recommended for adult cancer survivors, previous studies have focused only on the effects of aerobic exercise $[17,18]$. Besides, the prevalence of health behaviors and comorbidities such as obesity, smoking, alcohol, and cardiovascular diseases among cancer survivors have been reported in previous studies $[18,19]$. However, although these health behaviors and comorbidities may be associated with participation in PA, the data for these potential correlates of aerobic and resistance PA in cancer survivors are lacking.

To date, knowledge of the adherence to aerobic and resistance PA among cancer survivors and related factors, including age and sex, is limited. The aims of this study were to (1) assess the levels of engagement in aerobic and resistance exercises in cancer survivors, as stratified by age and sex, and (2) to identify the lifestyle or health-related correlates of meeting PA guidelines while adjusting for age and sex in Korean community-dwelling cancer survivors.

\section{METHODS}

\section{Study participants}

This study used cross-sectional data from the fourth, fifth, and sixth Korea National Health and Nutrition Examination Survey (KNHANES), which used a stratified, multistage, clustered probability sampling method to collect a representative sample of the civilian, community-dwelling Korean population. Household interviews and standardized physical examinations at mobile examination centers were performed. A total of 58,423 participants were recruited in the KNHANES from 2007 to 2013 with response rates between $71.2 \%$ and $82.8 \%$. The study population consisted of 39,845 participants $(16,871$ men and 22,974 women) aged $\geq 20$ years who completed a PA questionnaire and who underwent comprehensive health surveys and examinations. Participants who had ever been diagnosed with any cancer by a doctor were defined as cancer survivors $(n=1,254)[20,21]$. The Institutional Review Board at the Korea Centers for Disease Control and Prevention approved the protocol (IRB No. 2007-02CON-04-P, 2008-04EXP-01-C, 2009-01CON-032C, 2010-02CON-21-C, 2011-02CON-o6-C, 2012-01EXP01-2C, and 2013-07CON-03-4C), and all participants signed informed consent forms. Since this study used publicly available data, ethical approval of our hospital was not required.

\section{Physical activity assessment}

Aerobic PA was evaluated via the validated Korean version of the International Physical Activity Questionnaire Short Form (IPAQ-SF) [22-24]. The IPAQ-SF assesses PA during the last seven days of aerobic activities by measuring the frequency and duration of walking and other moderate-to-vigorous aerobic activity that was undertaken for more than ten continuous minutes across all contexts (i.e., work, transport, household, and leisure activities). By following the ACSM \& ACS PA guidelines for cancer survivors [12,13,25], the participants' aerobic exercise patterns were categorized as meeting recommendations for aerobic exercise (vigorous-intensity activity $\geq 75$ $\mathrm{min} / \mathrm{wk}$, moderate-intensity activity $\geq 150 \mathrm{~min} / \mathrm{wk}$ or an equivalent combination of the two) or as indicating an insufficient activity to meet recommendations for aerobic exercise. Because walking is regarded as a low-intensity activity, the time spent walking was not considered toward aerobic exercise. The KNHANES also recorded the number of days the participants engaged in resistance exercises such as push-ups, sit-ups, and exercises using dumbbells, weights, or iron bars during the last week. By following the ACSM PA guidelines, the participants' resistance exercise patterns were categorized as meeting recommendations for resistance exercise $(\geq 2$ day/wk of resistance exercise) or as indicating an insufficient activity to meet recommendations for resistance exercise.

\section{Clinical factors}

The participants of each sex were divided into three groups according to the following age groups: 20 to 49 
years (young), 50 to 64 years (middle-aged), and 65 years or older (old). Body mass index was calculated as each individual's weight divided by the square of the height $\left(\mathrm{kg} / \mathrm{m}^{2}\right)$. Smoking status (current, past, never), education level ( $\leq 9,>9$ years), and comorbidities (cardiovascular disease, diabetes, hypertension, and depression) were recorded. The mean daily alcohol intake was calculated from the questionnaires by recording the frequency of drinking days and the number of drinks consumed per drinking day. Heavy alcohol drinking was defined as $\geq$ $30 \mathrm{~g} /$ day for men and $\geq 20 \mathrm{~g} /$ day for women [26]. The questionnaires for cancer included the cancer site, current presence of cancer, current treatment for cancer, and age at diagnosis of cancer. Participants who were diagnosed with cancer $\geq 5$ years were defined as longterm cancer survivors.

\section{Statistical analysis}

The characteristics of the cancer survivors and noncancer controls were compared using the chi-square test and were presented as weighted percentages (standard errors of percentages). The age- and sex-stratified prevalence of meeting recommendations for ACSM and ACS aerobic and resistance PA guidelines in cancer survivors and noncancer controls was estimated by using complex-sample descriptive statistics and were compared using the chi-square test in each of the age- and sex-stratified subgroups. Odds ratios (ORs) with 95\% confidence intervals (CIs) for meeting PA recommendations were assessed by using multivariable-adjusted logistic regression analyses in cancer survivors to investigate the correlates of the adherences to aerobic and resistance exercise guidelines. Complex-samples logistic regression analyses were also employed to investigate the adherence to PA guidelines by cancer sites. Statistical analyses were performed by using the complex-sample procedures of SPSS software version 24 (IBM/SPSS Inc., Armonk, NY, USA). The weighted values during the stratification of the samples were reflected in all analyses. A p values $<0.05$ were considered statistically significant.

\section{RESULTS}

The weighted estimated prevalence of cancer survivors was $1.9 \%$ (standard error, $0.1 \%$ ) in the Korean population aged $\geq 20$ years. As shown in Table 1 , the percentages of participants who were old; were female; had low levels of education; or reported having cardiovascular disease, diabetes, hypertension, and depression were significantly higher in cancer survivors than in noncancer controls. In contrast, the percentages of participants who were current smokers and heavy alcohol drinkers were significantly lower in cancer survivors than in noncancer controls. The prevalence of meeting aerobic exercise guidelines was lower in cancer survivors, with a borderline significance. The prevalence of meeting resistance exercise guidelines was similar between cancer survivors and noncancer controls.

\section{Prevalence of meeting PA guidelines stratified by age and sex}

When the study population was stratified by age and sex, in males, the prevalence of meeting aerobic exercise recommendations in young, middle-aged, and old cancer survivors was $48.1 \%, 41.3 \%$, and $35.0 \%$, respectively (Fig. IA). In females, $46.4 \%$ of young cancer survivors, $39.3 \%$ of middle-aged cancer survivors, and $18.1 \%$ of old cancer survivors met the aerobic exercise recommendations. Female cancer survivors aged 20 to 49 years were significantly more likely to meet aerobic exercise recommendations than noncancer controls of the same age group and $\operatorname{sex}(p=0.003$ ) (Fig. 1A). Male cancer survivors aged $\geq 65$ years were more likely to meet aerobic exercise recommendations than noncancer controls of the same sex and age groups, with a borderline significance $(p=0.057)$ (Fig. 1A). In the other age and sex groups, the prevalence of meeting recommendations for aerobic exercise guidelines was similar between cancer survivors and noncancer controls.

When stratified by age and sex, in males, the prevalence of meeting resistance exercise guidelines in young, middle-aged, and old cancer survivors was $24.3 \%, 42.9 \%$, and $28.9 \%$, respectively (Fig. 1 B). In females, $14.0 \%$ of young cancer survivors, $16.9 \%$ of middle-aged cancer survivors, and $6.5 \%$ of old cancer survivors met the resistance exercise recommendations. The adherence to the resistance exercise guidelines was similar between cancer survivors and noncancer survivors in all age and sex groups. 
Table 1. Population characteristics

\begin{tabular}{|c|c|c|c|}
\hline Characteristic & $\begin{array}{c}\text { Cancer } \\
\text { survivors } \\
(\mathrm{n}=1,254)\end{array}$ & $\begin{array}{c}\text { Noncancer } \\
\text { controls } \\
(\mathrm{n}=38,591)\end{array}$ & $p$ value $^{a}$ \\
\hline Age, yr & & & $<0.001$ \\
\hline $20-49$ & $24.1(1.8)$ & $63.0(0.5)$ & \\
\hline $50-64$ & $36.5(1.8)$ & $23.5(0.3)$ & \\
\hline$\geq 65$ & $39.4(1.7)$ & $13.5(0.3)$ & \\
\hline Female sex & $61.2(1.8)$ & $50.3(0.3)$ & $<0.001$ \\
\hline $\mathrm{BMI}, \mathrm{kg} / \mathrm{m}^{2}$ & & & 0.052 \\
\hline$<23.0$ & $48.9(1.9)$ & $44.6(0.4)$ & \\
\hline $23.0-24.9$ & $22.4(1.5)$ & $23.3(0.3)$ & \\
\hline$\geq 25 \cdot 0$ & $28.7(1.7)$ & $32.1(0.3)$ & \\
\hline Current smoking & $7.8(1.0)$ & $25.5(0.3)$ & $<0.001$ \\
\hline $\begin{array}{l}\text { Heavy alcohol } \\
\text { drinking }\end{array}$ & $2.7(0.7)$ & $9.2(0.2)$ & $<0.001$ \\
\hline Low education, $\leq 9 \mathrm{yr}$ & $54.2(1.9)$ & $28.6(0.5)$ & $<0.001$ \\
\hline \multicolumn{4}{|l|}{ Comorbidities } \\
\hline $\begin{array}{l}\text { Cardiovascular } \\
\text { disease }\end{array}$ & $7.5(0.9)$ & $3.2(0.1)$ & $<0.001$ \\
\hline Diabetes & $15.1(1.3)$ & $8.5(0.2)$ & $<0.001$ \\
\hline Hypertension & $38.7(1.9)$ & $26.2(0.4)$ & $<0.001$ \\
\hline Depression & $6.3(0.9)$ & $3.3(0.1)$ & $<0.001$ \\
\hline $\begin{array}{l}\text { Long-term } \\
\text { survivor, } \geq 5 \text { yr }\end{array}$ & $54.3(1.9)$ & - & - \\
\hline $\begin{array}{l}\text { Current presence of } \\
\text { cancer }\end{array}$ & $43 \cdot 5(1.9)$ & - & - \\
\hline $\begin{array}{l}\text { Current treatment } \\
\text { for cancer }\end{array}$ & $35 \cdot 9(1.8)$ & - & - \\
\hline Cancer site & & & - \\
\hline Gastric & $20.5(1.5)$ & - & - \\
\hline Colorectal & $14.2(1.3)$ & - & - \\
\hline Thyroid & $10.8(1.2)$ & - & - \\
\hline Breast & $13.4(1.2)$ & - & - \\
\hline Cervical & $16.2(1.3)$ & - & - \\
\hline Prostate & $2.6(0.5)$ & - & - \\
\hline \multicolumn{4}{|l|}{$\begin{array}{l}\text { Meeting ACSM/ACS } \\
\text { guidelines }\end{array}$} \\
\hline Aerobic exercise & $36.2(1.7)$ & $39.7(0.4)$ & 0.053 \\
\hline Resistance exercise & $20.5(1.5)$ & $22.1(0.3)$ & 0.309 \\
\hline
\end{tabular}

Values are presented as weighted percentage (standard error of percentage).

BMI, body mass index; ACSM/ACS, the American College of Sports Medicine and American Cancer Society.

${ }^{a} p$ values by the chi-square test.
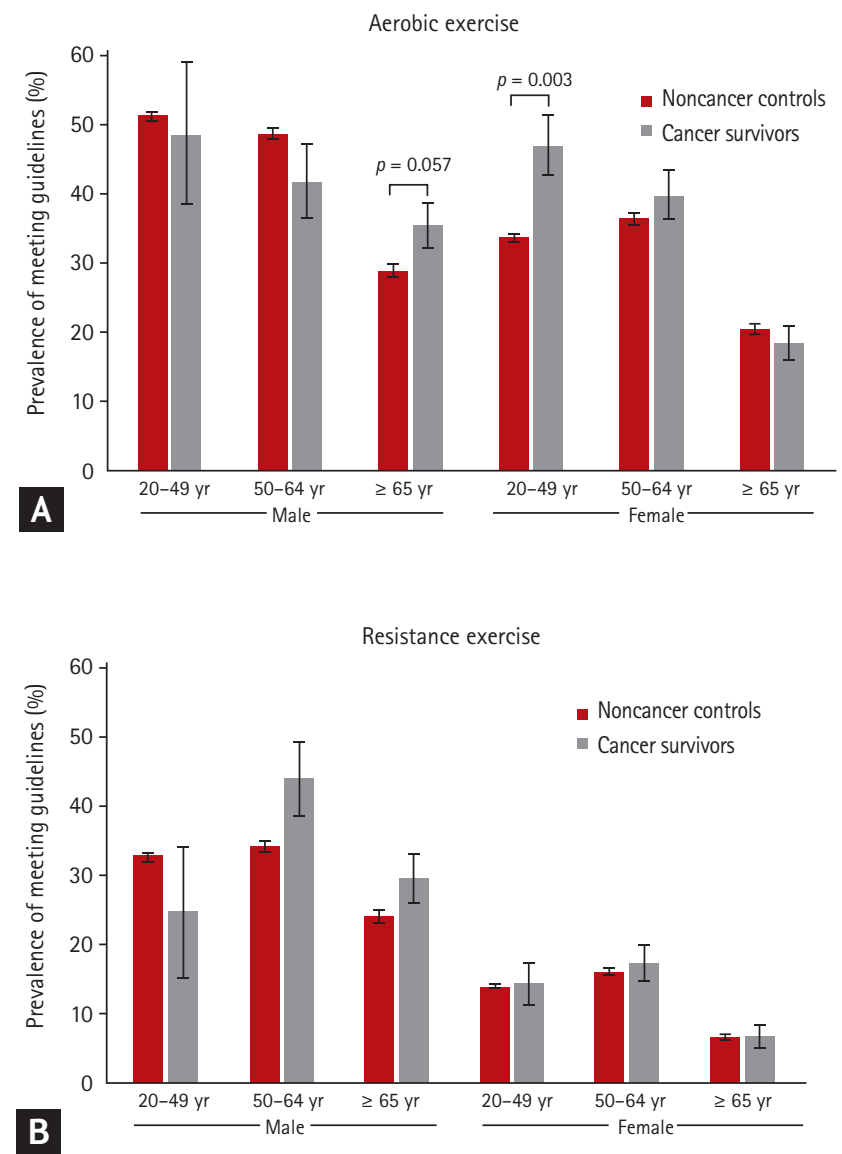

Figure 1. Age- and sex-stratified prevalence of meeting the recommendations for physical activity in cancer survivors and noncancer controls based on the American College of Sports Medicine and American Cancer Society (A) aerobic and (B) resistance exercise guidelines. Values are indicated as weighted percentages, and error bars represent the standard errors of the percentages.

\section{Correlates of meeting PA guidelines in cancer survivors}

The potential correlates of the adherence to the aerobic exercise guidelines, including age and sex, are shown in Table 2. The independent associations of age and sex with engagement in aerobic exercise were similar to the results of age- and sex-stratified analyses (Fig. 1A). Old cancer survivors were significantly less likely to engage in aerobic exercise at recommended levels than young adults, even after multivariable adjustments, including sex, health behaviors and comorbidities (adjusted OR, 0.44; $95 \%$ CI, 0.24 to 0.79 ). However, sex was not associated with adherence to aerobic exercise guidelines. Furthermore, in cancer survivors, being current smokers, having low education levels ( $\leq 9$ years), and having car- 
Table 2. Correlates of meeting the ACSM/ACS guidelines for aerobic and resistance exercises in cancer survivors ( $\mathrm{n}=1,254)$ : age, sex, sociodemographic, lifestyle, and clinical factors

\begin{tabular}{|c|c|c|c|c|}
\hline \multirow{2}{*}{ Variable } & \multicolumn{2}{|c|}{ Aerobic exercise } & \multicolumn{2}{|c|}{ Resistance exercise } \\
\hline & Unadjusted & Adjusted $^{\mathrm{a}}$ & Unadjusted & Adjusted $^{\mathrm{a}}$ \\
\hline \multicolumn{5}{|l|}{ Age, yr } \\
\hline $20-49$ & 1.00 & 1.00 & 1.00 & 1.00 \\
\hline $50-64$ & $0.76(0.49-1.17)$ & $0.82(0.49-1.37)$ & $1.79(1.02-3.14)^{b}$ & $2.00(1.02-3.92)^{b}$ \\
\hline$\geq 65$ & $0.40(0.26-0.61)^{b}$ & $0.44(0.24-0.79)^{b}$ & $1.03(0.58-1.83)$ & $0.26(0.60-2.65)$ \\
\hline Female sex & $0.80(0.59-1.09)$ & $0.69(0.46-1.02)$ & $0.29(0.20-0.42)^{b}$ & $0.32(0.21-0.50)^{b}$ \\
\hline \multicolumn{5}{|l|}{$\mathrm{BMI}, \mathrm{kg} / \mathrm{m}^{2}$} \\
\hline$<23.0$ & 1.00 & 1.00 & 1.00 & 1.00 \\
\hline $23.0-24.9$ & $0.93(0.63-1.36)$ & $0.98(0.65-1.47)$ & $1.38(0.89-2.13)$ & $1.14(0.69-1.89)$ \\
\hline$\geq 25.0$ & $0.93(0.65-1.33)$ & $0.90(0.59-1.38)$ & $0.72(0.47-1.13)$ & $0.82(0.49-1.39)$ \\
\hline Current smoking & $0.60(0.31-1.17)$ & $0.45(0.21-0.94)^{b}$ & $2.37(1.29-4.38)^{b}$ & $1.36(0.65-2.83)$ \\
\hline Heavy alcohol drinking & $0.71(0.24-2.15)$ & $0.29(0.07-1.16)$ & $1.67(0.47-5.94)$ & $0.67(0.09-4.95)$ \\
\hline Low education, $\leq 9 \mathrm{yr}$ & $0.49(0.36-0.67)^{b}$ & $0.68(0.47-0.99)^{b}$ & $0.51(0.35-0.74)^{b}$ & $0.47(0.29-0.74)^{b}$ \\
\hline Cardiovascular disease & $0.41(0.22-0.76)^{b}$ & $0.52(0.27-1.00)^{b}$ & $0.87(0.44-1.73)$ & $1.31(0.65-2.64)$ \\
\hline Diabetes & $0.79(0.50-1.23)$ & $1.05(0.65-1.70)$ & $0.87(0.51-1.48)$ & $0.81\left(0.45^{-1.45)}\right.$ \\
\hline Hypertension & $0.64(0.47-0.86)^{b}$ & $0.86(0.59-1.25)$ & $0.86(0.59-1.24)$ & $1.13(0.74-1.73)$ \\
\hline Depression & $1.18(0.64-2.17)$ & $1.45(0.74-2.87)$ & $0.99\left(0.45^{-2.18}\right)$ & $1.67(0.79-3.52)$ \\
\hline Long-term survivor, $\geq 5 \mathrm{yr}$ & $1.00(0.73-1.38)$ & $1.48(0.99-2.20)$ & $0.88(0.61-1.27)$ & $1.06(0.69-1.62)$ \\
\hline Current presence of cancer & $1.20(0.89-1.62)$ & $1.62(0.93-2.84)$ & $1.16(0.81-1.68)$ & $1.74(0.83-3.65)$ \\
\hline Current treatment for cancer & $1.04(0.75-1.43)$ & $0.66(0.36-1.21)$ & $0.96(0.66-1.42)$ & $0.64(0.29-1.39)$ \\
\hline
\end{tabular}

Values are presented as odds ratio (95\% confidence interval).

ACSM/ACS, the American College of Sports Medicine and American Cancer Society; BMI, body mass index.

${ }^{a}$ Adjusted for all other variables listed in the table.

${ }^{\mathrm{b}} \mathrm{A} p<0.05$ by complex-sample logistic regression models.

diovascular disease were associated with a significantly lower likelihood of meeting the recommendation for aerobic exercise after adjusting for age, sex, and other confounding variables.

Table 2 also shows the associations of age and sex with adherence to the resistance exercise guidelines, which were different from those of aerobic exercise guidelines. Middle-aged cancer survivors were more likely to meet the resistance exercise guidelines than young survivors (adjusted OR, 2.00; 95\% CI, 1.02 to 3.92). Females exhibited a significantly lower adherence to resistance exercise guidelines than males (adjusted OR,0.32; 95\% CI, 0.21 to 0.50). Except for age and sex, low education level was associated with a significantly lower likelihood of meeting the recommendations for resistance exercise in cancer survivors.

\section{Association of meeting PA guidelines with cancer site}

The prevalence of meeting the recommendations for PA guidelines was generally similar to that of noncancer controls in survivors of frequent cancer sites, except for in survivors of a few cancer sites (Fig. 2). Table 3 shows the adjusted ORs for meeting aerobic and resistance exercise guidelines of the frequent cancer survivors in the study population. The prostate cancer survivors were significantly more likely to meet the aerobic exercise guidelines than the noncancer controls after adjusting for age, smoking, education, and cardiovascular disease (adjusted OR, 2.93; 95\% CI, 1.42 to 6.05). Female colorectal cancer survivors exhibited a lower adherence to aerobic exercise guidelines than noncancer controls (unadjusted OR, 0.42; 95\% CI, o.19 to 0.92). However, adjustment for age, smoking, education, and cardiovascular disease 

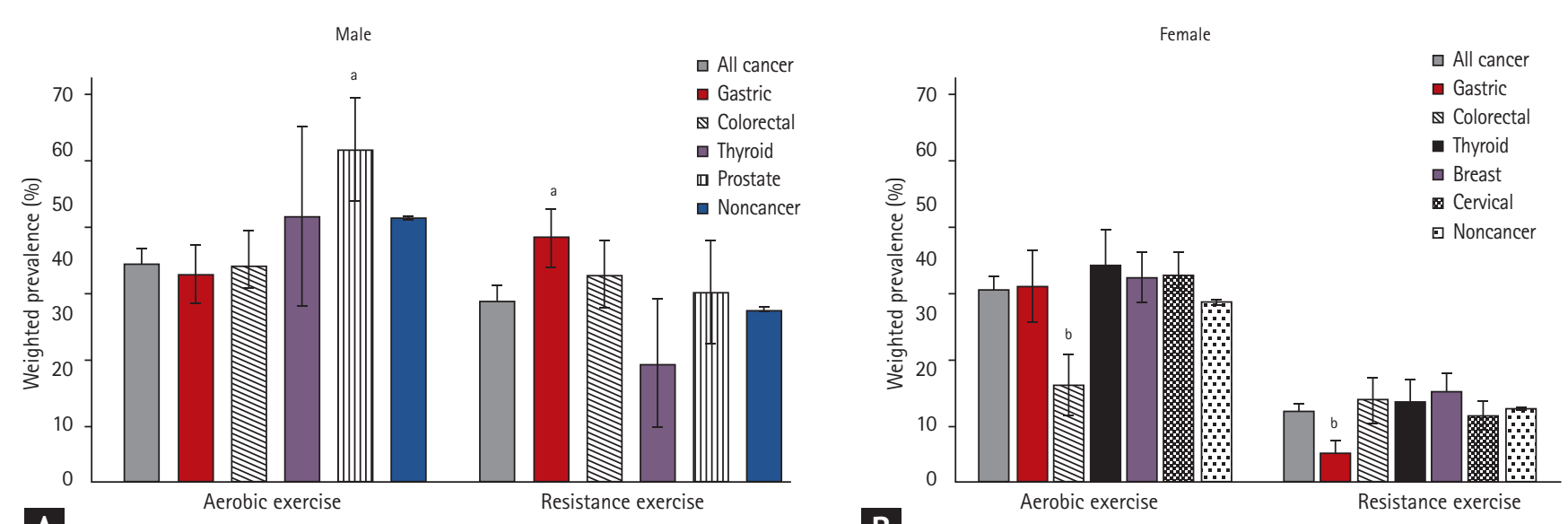

Figure 2. The prevalence of meeting the recommendations for aerobic and resistance exercise guidelines by cancer site in male (A) and female (B). Values are indicated as weighted percentages, and error bars represent the standard errors of the percentages. ${ }^{a} p<0.05$ by multivariable logistic regression analyses adjusted for age, smoking, education, and cardiovascular disease, noncancer controls as reference. ${ }^{b} p<0.05$ by univariable logistic regression analyses, noncancer controls as reference.

Table 3. Adjusted odds ratios for meeting the ACSM/ACS guidelines for aerobic and resistance exercises by cancer sites

\begin{tabular}{|c|c|c|c|c|}
\hline \multirow{2}{*}{ Variable } & \multicolumn{2}{|c|}{ Male } & \multicolumn{2}{|c|}{ Female } \\
\hline & Aerobic exercise $^{\mathrm{a}}$ & Resistance exercise $^{\mathrm{b}}$ & Aerobic exercise $^{\mathrm{a}}$ & Resistance exercise $^{b}$ \\
\hline Noncancer controls & 1.00 & 1.00 & 1.00 & 1.00 \\
\hline Gastric cancer & $1.00(0.64-1.55)$ & $2.05(1.29-3.26)^{c}$ & $1.38(0.79-2.43)$ & $0.37(0.12-1.14)$ \\
\hline Colorectal cancer & $1.00\left(0.55^{-1.82}\right)$ & $1.46(0.81-2.62)$ & $0.55(0.26-1.18)$ & $1.60(0.86-3.00)$ \\
\hline Thyroid cancer & $1.03(0.23-4.50)$ & $0.52(0.13-2.12)$ & $1.38(0.80-2.37)$ & $1.13(0.56-2.27)$ \\
\hline Prostate cancer & $2.93(1.42-6.05)^{c}$ & $1.23(0.52-2.93)$ & - & - \\
\hline Breast cancer & - & - & $1.34(0.91-1.97)$ & $1.37(0.82-2.28)$ \\
\hline Cervical cancer & - & - & $1.37(0.96-1.95)$ & $0.98(0.58-1.65)$ \\
\hline$p$ for trend & 0.077 & 0.006 & 0.119 & 0.321 \\
\hline
\end{tabular}

Values are presented as odds ratio (95\% confidence interval).

ACSM/ACS, the American College of Sports Medicine and American Cancer Society.

${ }^{a}$ Adjusted for age, smoking, education, and cardiovascular disease.

${ }^{\mathrm{b}}$ Adjusted for age and education.

${ }^{\mathrm{c}} \mathrm{A} p<0.05$ by complex-sample logistic regression models.

nullified this association (adjusted OR, 0.55; 95\% CI, 0.26 to 1.18). Male gastric cancer survivors were more likely to engage in resistance exercises than noncancer controls (unadjusted OR, 1.79; 95\% CI, 1.15 to 2.78 ); female gastric cancer survivors were less likely to engage in resistance exercises than noncancer controls (unadjusted OR, 0.32; $95 \% \mathrm{CI}$, 0.10 to 0.97 ). This association remained significant in males (adjusted OR, 2.05; 95\% CI, 1.29 to 3.26) but became insignificant in females after adjustment for age and education (adjusted OR, 0.37; 95\% CI, 0.12 to 1.14).
The prevalence of frequent cancer sites according to age and sex is shown in Supplementary Fig. 1.

\section{DISCUSSION}

During the past few decades, the importance of PA in cancer survivors has been emphasized, and substantial efforts have been made to increase exercise compliances $[12,13]$. However, the results of this study demonstrated 
that only less than half of community-dwelling cancer survivors met aerobic exercise guidelines and that less than a quarter of cancer survivors met resistance exercise guidelines. When comparing the adherence to aerobic and resistance exercise guidelines between cancer survivors and controls who did not have a history of cancer, young female cancer survivors were more likely to meet aerobic exercise guidelines than noncancer female controls in the same age group. Old male cancer survivors tended to be more engaged in aerobic exercise than noncancer controls of the same age group and sex. The independent correlates of exercise adherence in aerobic exercise were age, smoking, education, and cardiovascular disease, and those in resistance exercise were age, sex, and education. Among the cancer survivors, the elderly individuals participated less in aerobic exercise than the young individuals, and female cancer survivors performed fewer resistance exercises than male survivors. The cancer survivors who were current smokers or had cardiovascular diseases were less likely to meet aerobic exercise guidelines. Prostate cancer survivors exhibited a higher likelihood of meeting aerobic exercise guidelines than noncancer controls. Male gastric cancer survivors were more likely to engage in resistance exercise with the recommended level.

Although the importance of PA in old cancer survivors has been reported to prevent functional decline [27], the adherence to aerobic PA in old cancer survivors was significantly lower than in young cancer survivors, even after adjustments for health behaviors and comorbidities. The lower prevalence of meeting aerobic exercise guidelines in old cancer survivors was in agreement with a previous report from the 2014 National Health Interview Survey in the United States [15]. Old cancer survivors have difficulty in engaging in moderate-to-vigorous PA because they have more pain or disabilities. Furthermore, there are no detailed guidelines for the optimal intensity, mode, or duration of PA specifically developed for old cancer survivors $[12,13,25]$. Therefore, old cancer survivors may have difficulty in determining exercise methods and maintaining exercise for an extended period. Future studies are needed to provide age group specific guidelines for cancer survivors, especially for frail, old cancer survivors.

There is a controversy regarding the difference in aerobic PA levels between cancer survivors and the gener- al population without a cancer history. Several studies have observed similar aerobic PA engagement levels between cancer survivors and noncancer controls [15,18,28]. Conversely, other studies have reported lower levels of aerobic PA in cancer survivors than in noncancer controls [29,30]. The current study demonstrated that the prevalence of meeting aerobic exercise guidelines in all cancer survivors tended to be lower than in noncancer controls. However, when all cancer survivors were stratified according to age and sex, the aerobic PA engagement was significantly higher in young female cancer survivors and predisposed to be higher in old male cancer survivors than in noncancer controls of the same age and sex. The higher prevalence of meeting aerobic PA guidelines in young female and old male cancer survivors might be a notably meaningful finding. Some cancer survivors may engage in exercise to improve their health without recognizing themselves as being vulnerable and unable to exercise.

In addition to age and sex, which are primitive, unmodifiable factors, a current smoking status, education status, and cardiovascular disease status were independent correlates of adherence to aerobic exercise guidelines after adjustments for age and sex. The current study results, which indicated that smoking was independently associated with the adherence to the aerobic exercise guidelines but not with the adherence to resistance exercise guidelines, suggest that smoking cessation would be an important health intervention in promoting aerobic exercise engagement in cancer survivors. Cancer survivors have better treatment outcomes if they quit smoking [31]. There can be a vicious cycle in which comorbid cardiovascular disease may lead to aerobic inactivity, and aerobic inactivity may increase the risk of cardiovascular disease in cancer survivors. When considering the vicious cycle and the fact that cardiovascular disease is the leading cause of noncancer deaths among cancer survivors [32,33], attention should be paid to increasing levels of aerobic activity in cancer survivors with cardiovascular disease.

Despite the numerous beneficial effects of resistance exercise and this type of exercise being recommended in cancer survivors $[12,13,32,33]$, the adherence to resistance exercise guidelines has been investigated relatively less than that to aerobic exercise guidelines. The weighted prevalence of meeting resistance exercise guidelines in 
overall Korean cancer survivors from this study $(20.5 \% \pm$ $1.5 \%)$ was comparable to that of previous studies $[15,16,34]$. Interestingly, the proportion of female cancer survivors who met the resistance exercise guidelines was low in all age groups and was lower than the proportions reported in previous studies [16,34]. A possible explanation is the effects of sociocultural factors, such as preference for exercise types and accessibility to resistance exercise in Korean female, when considering the consistent low adherence to resistance exercise guidelines in females without a cancer history. The noticeably lower participation in resistance exercise of female cancer survivors than of male cancer survivors with the same cancer site also supports this explanation of sociocultural factors.

Given that the adherence to resistance exercise guidelines in non-hospitalized cancer survivors of all age and sex groups was not higher than in people without cancer, it is presumed that the importance of resistance exercise in cancer survivors might not have been well taught to the survivors. Although behavioral counseling by health care providers has been reported to promote PA [35], oncologists recommended resistance exercise less frequently than aerobic exercise in a previous study [36]. Therefore, encouraging resistance exercise as well as aerobic exercise in cancer survivors by health care providers should be emphasized.

Although the analysis of PA engagement according to cancer sites is important, previous epidemiological studies have reported limited results with a different classification of cancer sites across studies [16-18]. This heterogeneity may originate from the numerous cancer sites [3], a different profile of frequent cancers across country and race. In the present study, prostate cancer survivors showed a higher likelihood of meeting aerobic PA guidelines than noncancer controls. Male gastric cancer survivors showed higher adherence to the resistance exercise guidelines than noncancer controls. The differences in the adherence to aerobic and resistance exercise guidelines of cancer survivors with the same cancer site suggested that sex may affect the PA of cancer survivors. A previous large cross-sectional study reported higher aerobic PA levels among prostate cancer survivors and lower PA levels among middle-aged cervical and endometrial cancer survivors, than those among sex- and age group-matched general populations [17]. Another study found that the likelihood of meeting re- sistance training and aerobic activity guidelines was significantly lower for women with a history of any cancer, except breast cancer, than for women with no history of cancer [16]. However, it seems arbitrary to classify the cancer sites as binary variables into breast cancer and all other cancers.

The strength of the current study is that we used nationally representative data from community-dwelling cancer survivors. Besides, age- and sex-stratified prevalence and age- and sex-adjusted correlates would provide valuable information to increase the engagement in aerobic and resistance exercises in cancer survivors. Nevertheless, there are several limitations to this study. First, PA was evaluated by self-reported questionnaires with a risk of recall bias. There were no objective measurements (e.g., accelerometer or pedometer) of PA in KNHANES. Second, it was impossible to assess the direction of causality due to the inherent limitation of the cross-sectional design. Although the elapsed time since cancer diagnosis can be an important factor influencing PA in cancer survivors [37,38], the cross-sectional design also prevented the analysis of the effect of time since cancer diagnosis. Third, there was no detailed information about cancer, such as cancer stage and treatment modalities. Fourth, since the incidence of cancer, prevalent cancer sites, and sociocultural factors vary across each country, this study results may not be generalized to other countries. Finally, because the sample size of the cancer survivors in the current study was modest, the analysis regarding the association of age, sex, and other factors with PA in each cancer site subgroups was not possible.

In conclusion, cancer survivors showed low participation in PA, which was associated with age, sex, and other sociodemographic and clinical factors. Old age, current smoking status, low education status, and concomitant cardiovascular disease were associated with lower adherence to aerobic exercise guidelines, whereas female sex and low education status were associated with lower adherence to resistance exercise guidelines. These results may help clinicians recognize cancer survivors who are less likely to participate in PA and encourage aerobic and resistance exercises for this population. Further studies will be required to identify the appropriate exercise prescriptions when considering age, sex, and other correlates. 


\section{KEY MESSAGE}

1. Only $36.2 \%$ of community-dwelling cancer survivors met aerobic exercise guidelines, and $20.5 \%$ of cancer survivors met resistance exercise guidelines.

2. Old cancer survivors engaged less in aerobic exercise, and female cancer survivors participated less in resistance exercises.

3. Smoking and cardiovascular disease were independent negative correlates of aerobic exercise engagements among cancer survivors.

\section{Conflict of interest}

No potential conflict of interest relevant to this article was reported.

\section{REFERENCES}

1. Cancer survivors: living longer, and now, better. Lancet 2004;364:2153-2154.

2. Jung KW, Won YJ, Kong HJ, Lee ES; Community of Population-Based Regional Cancer Registries. Cancer statistics in Korea: incidence, mortality, survival, and prevalence in 2015. Cancer Res Treat 2018;50:303-316.

3. Siegel RL, Miller KD, Jemal A. Cancer statistics, 2017. CA Cancer J Clin 2017;67:7-30.

4. Annunziata MA, Muzzatti B, Flaiban C, et al. Long-term quality of life profile in oncology: a comparison between cancer survivors and the general population. Support Care Cancer 2018;26:651-656.

5. Ng HS, Roder D, Koczwara B, Vitry A. Comorbidity, physical and mental health among cancer patients and survivors: an Australian population-based study. Asia Pac J Clin Oncol 2018;14:e181-e192.

6. Ogle KS, Swanson GM, Woods N, Azzouz F. Cancer and comorbidity: redefining chronic diseases. Cancer 2000;88:653-663.

7. De Groef A, Penen F, Dams L, van der Gucht E, Nijs J, Meeus M. Best-evidence rehabilitation for chronic pain part 2: pain during and after cancer treatment. J Clin Med 2019;8:E979.

8. Cramp F, Byron-Daniel J. Exercise for the management of cancer-related fatigue in adults. Cochrane Database Syst
Rev 2012;11:CDoo6145.

9. Fong DY, Ho JW, Hui BP, et al. Physical activity for cancer survivors: meta-analysis of randomised controlled trials. BMJ 2012;344:e70.

10. Mishra SI, Scherer RW, Geigle PM, et al. Exercise interventions on health-related quality of life for cancer survivors. Cochrane Database Syst Rev 2012;8:CDoo7566.

11. Gunnell AS, Joyce S, Tomlin S, et al. Physical activity and survival among long-term cancer survivor and non-cancer cohorts. Front Public Health 2017;5:19.

12. Schmitz KH, Courneya KS, Matthews C, et al. American College of Sports Medicine roundtable on exercise guidelines for cancer survivors. Med Sci Sports Exerc 2010;42:1409-1426.

13. Rock CL, Doyle C, Demark-Wahnefried W, et al. Nutrition and physical activity guidelines for cancer survivors. CA Cancer J Clin 2012;62:243-274.

14. Katzmarzyk PT, Lee IM, Martin CK, Blair SN. Epidemiology of physical activity and exercise training in the United States. Prog Cardiovasc Dis 2017;60:3-10.

15. Tarasenko Y, Chen C, Schoenberg N. Self-reported physical activity levels of older cancer survivors: results from the 2014 National Health Interview Survey. J Am Geriatr Soc 2017;65:e39-e44.

16. Ottenbacher A, Yu M, Moser RP, Phillips SM, Alfano C, Perna FM. Population estimates of meeting strength training and aerobic guidelines, by gender and cancer survivorship status: findings from the Health Information National Trends Survey (HINTS). J Phys Act Health 2015;12:675-679.

17. Kwon S, Hou N, Wang M. Comparison of physical activity levels between cancer survivors and non-cancer participants in the 2009 BRFSS. J Cancer Surviv 2012;6:54-62.

18. Coups EJ, Ostroff JS. A population-based estimate of the prevalence of behavioral risk factors among adult cancer survivors and noncancer controls. Prev Med 2005;40:702711.

19. Pinto BM, Eakin E, Maruyama NC. Health behavior changes after a cancer diagnosis: what do we know and where do we go from here? Ann Behav Med 2000;22:38-52.

20. Deimling GT, Bowman KF, Wagner LJ. Cancer survivorship and identity among long-term survivors. Cancer Invest 2007;25:758-765.

21. Mayer DK, Nasso SF, Earp JA. Defining cancer survivors, their needs, and perspectives on survivorship health care in the USA. Lancet Oncol 2017;18:e11-e18. 
22. Craig CL, Marshall AL, Sjostrom M, et al. International physical activity questionnaire: 12-country reliability and validity. Med Sci Sports Exerc 2003;35:1381-1395.

23. Oh JY, Yang YJ, Kim BS, Kang JH. Validity and reliability of Korean version of International Physical Activity Questionnaire (IPAQ) short form. J Korean Acad Fam Med 2007;28:532-541.

24. Fogelholm M, Malmberg J, Suni J, et al. International Physical Activity Questionnaire: validity against fitness. Med Sci Sports Exerc 2006;38:753-760.

25. American College of Sports Medicine. ACMS's Guidelines for Exercise Testing and Prescription. 1oth ed. Philadelphia (PA): Lippincott Williams \& Wilkins, 2017.

26. Chalasani N, Younossi Z, Lavine JE, et al. The diagnosis and management of nonalcoholic fatty liver disease: practice guidance from the American Association for the Study of Liver Diseases. Hepatology 2018;67:328-357.

27. Klepin HD, Mohile SG, Mihalko S. Exercise for older cancer patients: feasible and helpful? Interdiscip Top Gerontol 2013;38:146-157.

28. Oh MG, Han MA, Park J, Ryu SY, Park CY, Choi SW. Health behaviors of cancer survivors: the fourth Korea National Health and Nutrition Examination Survey (KNHANES IV, 2007-09). Jpn J Clin Oncol 2013;43:981-987.

29. Mayer DK, Terrin NC, Menon U, et al. Health behaviors in cancer survivors. Oncol Nurs Forum 2007;34:643-651.

30. Fairley TL, Hawk H, Pierre S. Health behaviors and quality of life of cancer survivors in Massachusetts, 2006: data use for comprehensive cancer control. Prev Chronic Dis
2010;7:A09.

31. Hymowitz N. Smoking and cancer: a review of public health and clinical implications. J Natl Med Assoc 2011;103:695-700.

32. Strasser B, Steindorf K, Wiskemann J, Ulrich CM. Impact of resistance training in cancer survivors: a meta-analysis. Med Sci Sports Exerc 2013;45:2080-2090.

33. Focht BC, Clinton SK, Devor ST, et al. Resistance exercise interventions during and following cancer treatment: a systematic review. J Support Oncol 2013;11:45-6o.

34. Forbes CC, Blanchard CM, Mummery WK, Courneya K. Prevalence and correlates of strength exercise among breast, prostate, and colorectal cancer survivors. Oncol Nurs Forum 2015;42:118-127.

35. Tarasenko YN, Miller EA, Chen C, Schoenberg NE. Physical activity levels and counseling by health care providers in cancer survivors. Prev Med 2017;99:211-217.

36. Karvinen KH, DuBose KD, Carney B, Allison RR. Promotion of physical activity among oncologists in the United States. J Support Oncol 2010;8:35-41.

37. Irwin ML, Crumley D, Mc'Tiernan A, et al. Physical activity levels before and after a diagnosis of breast carcinoma: the health, eating, activity, and lifestyle (HEAL) study. Cancer 2003;97:1746-1757.

38. Fassier P, Zelek L, Partula V, et al. Variations of physical activity and sedentary behavior between before and after cancer diagnosis: results from the prospective population-based NutriNet-Sante cohort. Medicine (Baltimore) 2016;95:e4629. 

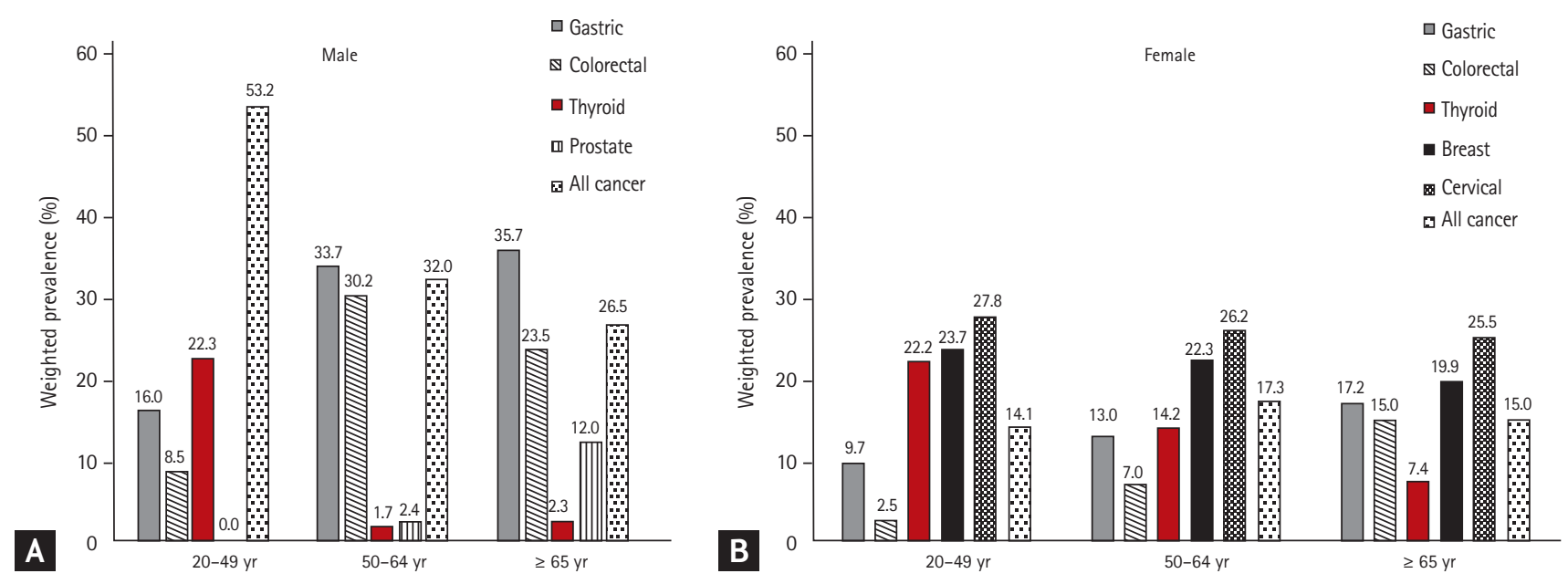

Supplementary Figure 1. Prevalence of cancer site according to age in male (A) and female (B). Values are indicated as weighted percentages. 